LBL- 19810

SSC Mag Note-47

\title{
THE USE OF POISSON TO CALCULATE THE EFFECT OF MAGNETIZATION IN SUPERCONDUCTING MAGNETS*
}

S. Caspi, W.S. Gilbert, M. Helm, and L.J. Laslett

Lawrence Berkeley Laboratory

University of Callfornia

Berkeley, CA 94720

August 1985

*This work was supported by the Director, Office of Energy Research, Office of High Energy and Nuclear Physics, High Energy Physics Division, U.S. Dept. of Energy, under Contract No. DE-ACO3-76SF00098. 


\title{
THE USE OF POISSON TO CALCULATE THE EFFECT OF MAGNETIZATION IN SUPERCONDUCTING MAGNETS*
}

\author{
S. Caspi, W.S. Gilbert, M. Helm, and L.J. Laslett \\ Lawrence Berkeley Laboratory \\ University of California \\ Berkeley, CA 94720
}

\section{INTRODUCTION}

Magnetostatic problems solved by POISSON employ current and air regions as well as regions of nonlinear permeable iron. In many problems it is customary to set the permeability of the current regions identical to that of air and to introduce a permeability table (e.g. B-H) for the iron regions.' If the conductor is made of a superconducting material, setting the permeability of the current regions equal to that of air ls only an approximation. The existence of surface and bulk super-currents, which act partially to shield the superconductor's interior from the penetrating field, results in the superconductor acquiring a magnetization that in some cases cannot be ignored. Magnetization in superconducting dipole magnets influences the field uniformity. This effect is quite small at high fields ( $\mathrm{H} \gg \mathrm{Hp}$; $\mathrm{Hp}=$ fleld at penetration) but introduces large harmonic coefficlents at low flelds where the magnitude of the magnetization is of the order of the applied field.

Magnetization of a superconducting material can be introduced into POISSON through a field dependent permeability table (in much the same way that iron characteristics are introduced). This can be done by representing the increasing and decreasing field characteristics by two independent magnetization curves. We have verifled that superposition of a current and a magnetization table in the same region does not violate the code.

"This was supported by the Director, Office of Energy Research, Office of High Energy and Nuclear Physics, High Energy Physics Division, U.S. Dept. of Energy, under Contract No. DE-AC03-76SF00098. 
A similar method for calculating magnetization effects was recently proposed by M. Kuchnir and E. Fisk at Fermilab. However, the method outlined here takes advantage of integrating magnetization effects into the field relaxation process and thereby avoiding some of the inaccuracies introduced by perturbation techniques. G. Morgan of BNL also has reported on the use of GFUN to calculate magnetization effects using a similar method.

We present here two examples. The first uses a linear and reversible magnetization curve for which an analytical solution is compared with that of

POISSON. The second is a more realistic case where a measured magnetization curve of a superconducting cable is introduced into POISSON and results are compared with measurements.

\section{EXAMPLE I - MAGNETIZATION OF A CURRENT CARRYING ANNULUS}

\section{Analytical Solution}

We first analyze an arrangement (sketched below) in which a current I flows, with constant current density, into an annulus of inner and outer radil $a, b$ and returns as Io through the annulus center.

The $2 D$ ring cross section area is:

$$
A=\pi\left(b^{2}-a^{2}\right)
$$

The current density is:

$$
J_{0}=\frac{I_{0}}{\pi\left(b^{2}-a^{2}\right)}
$$

We make use of relations:

$$
\begin{aligned}
& \int H d l=\frac{4 \pi}{10} I \\
& B=\mu_{r} H
\end{aligned}
$$




$$
\begin{aligned}
& H=\text { Oersted } \\
& \ell=\mathrm{cm} \\
& I=\text { Amp } \\
& B=\text { Gauss } .
\end{aligned}
$$

Then, for $r<a$,

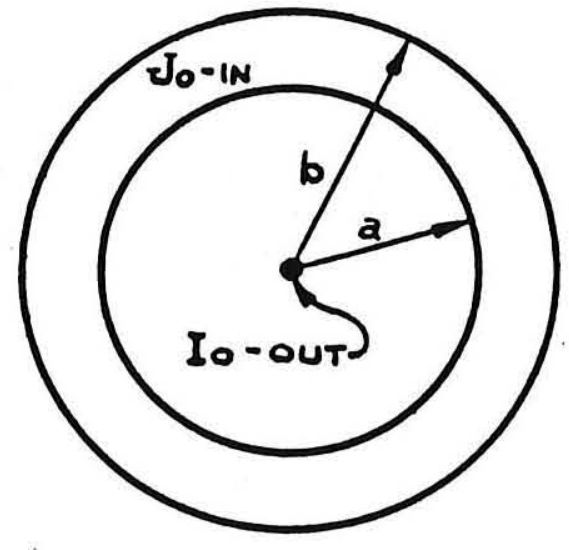

$$
\text { Then, for } r<a \text {, }
$$

$$
2 \pi r H_{\theta}=\frac{4 \pi}{10} I_{0}
$$

$$
H_{\theta}=\frac{2 I}{10 r}
$$

For $b>r>a, \quad I=I_{0}-J_{0} \pi\left(r^{2}-a^{2}\right)=I_{0} \frac{b^{2}-r^{2}}{b^{2}-a^{2}}$

$$
\begin{gathered}
2 \pi r H_{\theta}=\frac{4 \pi}{10} I_{0} \frac{b^{2}-r^{2}}{b^{2}-a^{2}} \\
H_{\Theta}=\frac{2 I_{0}}{10} \frac{b^{2}-r^{2}}{r\left(b^{2}-a^{2}\right)} \\
B_{\theta}=\frac{2 I_{0}}{10} \mu_{r} \frac{b^{2}-r^{2}}{r\left(b^{2}-a^{2}\right)} ;
\end{gathered}
$$

and for $r>b$,

$$
H_{\theta}=0 \text {. }
$$

If $\mu_{r} \neq 1$ in the ring, then $B \neq H$ - corresponding to a magnetization curve sketched below: 


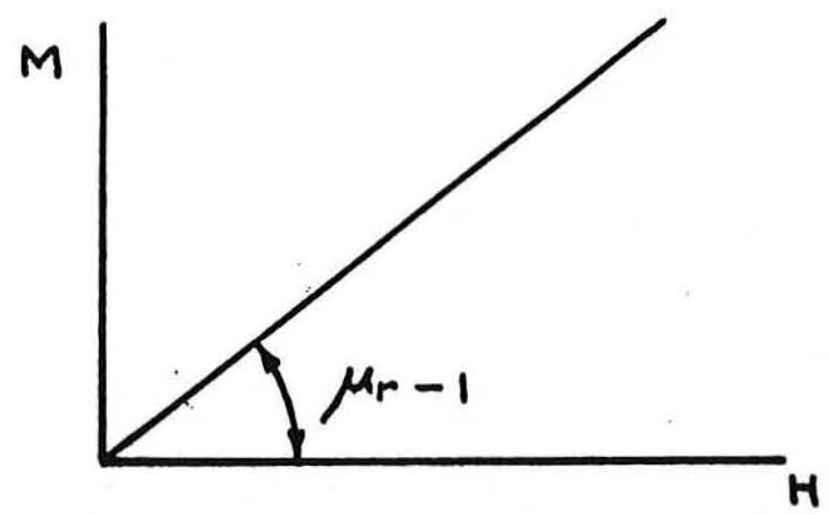

- We now derive the vector potential $A_{1}$ using $B_{\theta}=-\partial A / \partial r$, so that it can be compared directly with POISSON's output.

For $r>b$ : $\quad$ Since $A=$ constant, we choose $A=0$.

For $b>r>a$ :

$$
\begin{gathered}
A=\frac{2 I_{0}}{10} \mu_{r} \int_{r}^{b} \frac{b^{2}-r^{2}}{r\left(b^{2}-a^{2}\right)} d r \\
A=\frac{2 I_{0}}{10} \mu_{r}\left[\frac{b^{2}}{b^{2}-a^{2}} \ln \frac{b}{r}-\frac{1}{2} \frac{b^{2}-r^{2}}{b^{2}-a^{2}}\right] \\
A=\frac{2 I_{0}}{10} \mu_{r}\left[\frac{b^{2}}{b^{2}-a^{2}} \ln \frac{b}{a}-\frac{1}{2}\right] .
\end{gathered}
$$

and on $r=a$

For $r<a$ :

$$
\begin{gathered}
A=A_{r=a}-\frac{2 I 0}{10} \ln \frac{r}{a} \\
A=\frac{2 I 0}{10}\left[\mu_{r}\left(\frac{b^{2}}{b^{2}-a^{2}} \ln \frac{b}{a}-\frac{1}{2}\right)-\ln \frac{r}{a}\right] .
\end{gathered}
$$

If we select $a=1 \mathrm{~cm}, b=2 \mathrm{~cm}$, and $I_{0}=4000$ Amp, we calculate: 


$$
\begin{array}{cc}
r \geqslant 2 & A=0 \\
1 \leqslant r \leqslant 2 & A=800 \mu_{r}\left[\frac{4}{3} \ln \frac{2}{r}-\frac{4-r^{2}}{6}\right] \\
r \leqslant 1 & A=800\left[\mu_{r} 0.424196-\ln r\right]
\end{array}
$$

In Table I below we compare numerical results for $\mu_{r}=0.5$ and 1.5.

\begin{tabular}{c|c|c|c|c}
$\mu_{r}$ & $r(\mathrm{~cm})$ & $A-$ analytical & $A-$ Poisson & $\Delta \%$ \\
\hline \multirow{2}{*}{0.5} & 0.5 & 724.20 & 723.3 & 0.12 \\
& 1.0 & 169.68 & 169.28 & 0.24 \\
& 1.5 & 36.76 & 36.6 & 0.45 \\
& & & & \\
1.5 & 0.5 & 1063.55 & 1062.3 & 0.12 \\
& 1.0 & 509.04 & 508.15 & 0.17 \\
& 1.5 & 110.29 & 110.0 & 0.26
\end{tabular}

\section{EXAMPLE 2 - MAGNETIZATION OF A SUPERCONDUCTING DIPOLE MAGNET}

\section{Method and Application}

We construct two groups of input tables for POISSON, intended to describe the magnetization of superconductor cables used in a dipole magnet [D-12C-2]. One group of tables includes all magnetization curves, of various cable types, during a field increase and the other provides similar curves for a decreasing field.

We require magnetization curves for the identical cables used in this magnet in order to take care of variations in strand size, copper to superconductor ratio, transport current, and critical current. The magnetization curve of an entire block and not of a single turn (or cable) will be required to take care of insulation, cable compactness, 
small wedges, and other non-magnetic materials, since current regions in POISSON are usually represented by a single block rather than by a collection of individual turns.

In many cables measured magnetization curves may not be available and then the use of scaling may be required. The magnetization curve should be avallable over a range of field extending to values as high as the short-sample limit.

\section{Magnet D-12C-2}

We next compare the measured sextupole and decapole moments of LBL-SSC - model magnet D-12C-2 with those calculated by POISSON.

The inner and outer layers of the 4-cm bore two-layer magnet (Fig. 1) are made of a 23-strand and a 30-strand cable respectively, with 1.3 and $1.8 \mathrm{Cu} / \mathrm{sc}$ ratios. Stainless-steel collars over the outer layer displaced the iron to a radius of $5.57 \mathrm{~cm}$. We have ignored possible saturation of the iron and therefore set the iron permeability to $\mu=\infty$ in these studies of magnetization effects. It is planned that the effect of images in iron of variable permeabilities will be checked in later work. Each individual layer has been subdivided in the computations into two parts of equal radial thickness in order to take care of the radial dependency of the current density and magnetization.

At the time this work was carried out only magnetization measurements for the inner layer were available to us. Such data took into account the existence of copper and superconductor only. We therefore took the steps necessary to scale this single magnetization curve so as to reflect the physical conditions in each of the sublayers as they exist during magnet operation. The full details of the calculations have been placed in Appendix A.

\section{Results}

A series of POISSON runs was made (total of 32 ) to produce data in the range of $0.1 \mathrm{~T}$ to $6.8 \mathrm{~T}$. The first half of the runs employed magnetization tables corresponding 


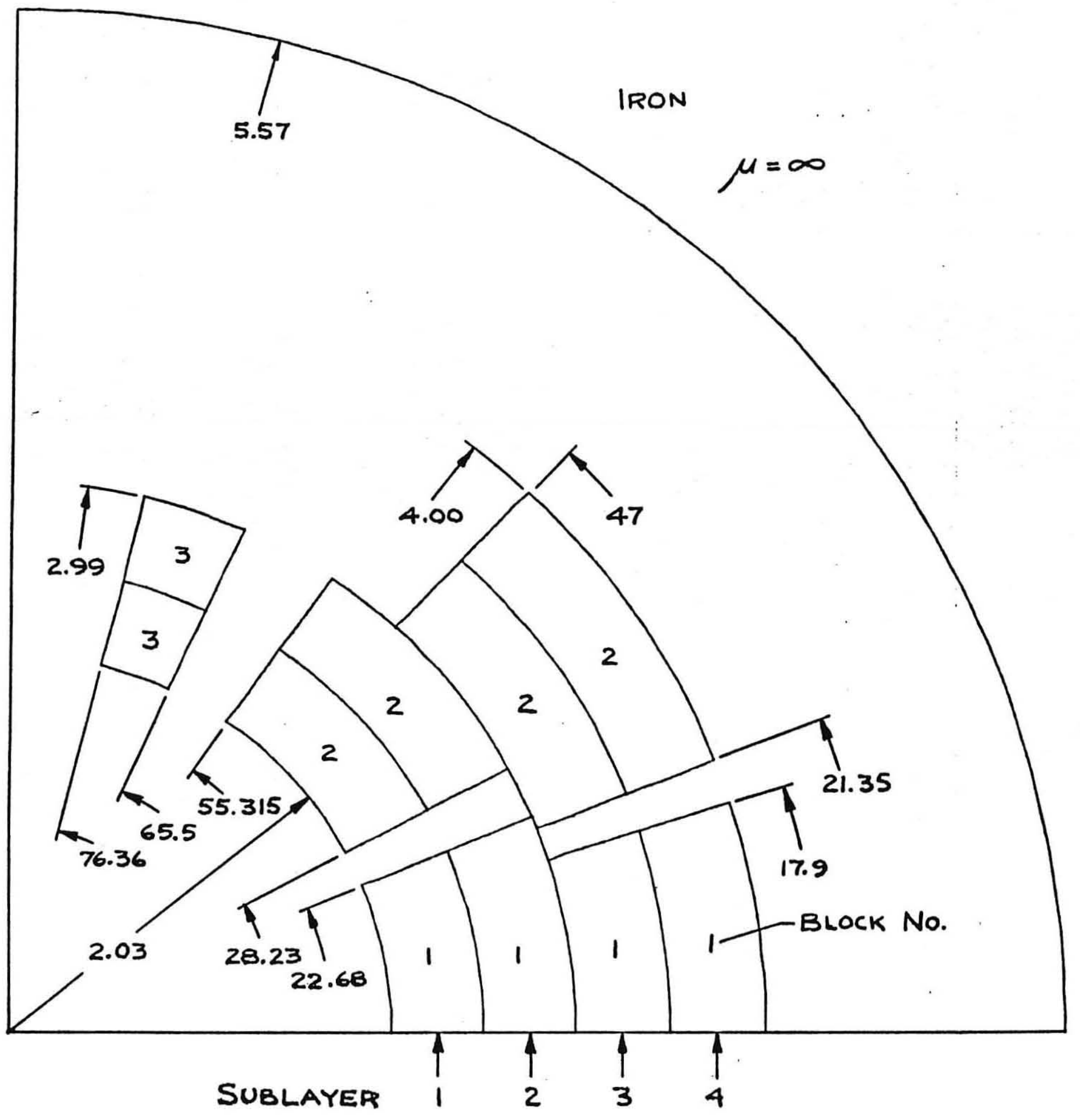

Fig. 1. Two-layer SSC type dipole used in calculations of magnetization. Numbers in current blocks correspond to the index scheme used in Appendix A. 
to an increase in current, and the remainder, for the same fleld interval, employed magnetization tables for decreasing current. At each fleld level we obtained two solutions such that upon subtracting their vector potential values we were left with a vector potential that corresponds to the total field change due to magnetization effects. The differential field harmonics (up minus down) were calculated and are plotted in Figs. 2-4. The individual sextupole and decapole coefficients for a full cycle $\left(b_{2}\right.$ and $\left.b_{4}\right)$ are plotted in Figs. 5 and 6 (all harmonic calculations were performed at $1 \mathrm{~cm}$ radius). Flux lines during a current increase at $0.28 \mathrm{~T}$ are plotted in Fig. 7. The - effect of magnetization for these two curves can scarcely be distinguished. In order to suppress the dominating transport current effect we accordingly have calculated the fleld assuming no magnetization ( $\mu=1$ in the current regions), and then subtracted the resulting vector potential from the vector potential at the same field when magnetization is present. Flux lines due to magnetization only are plotted in Fig. 8. 
$\Delta$ Dipole for design $\mathrm{D}(4 \mathrm{~cm})$

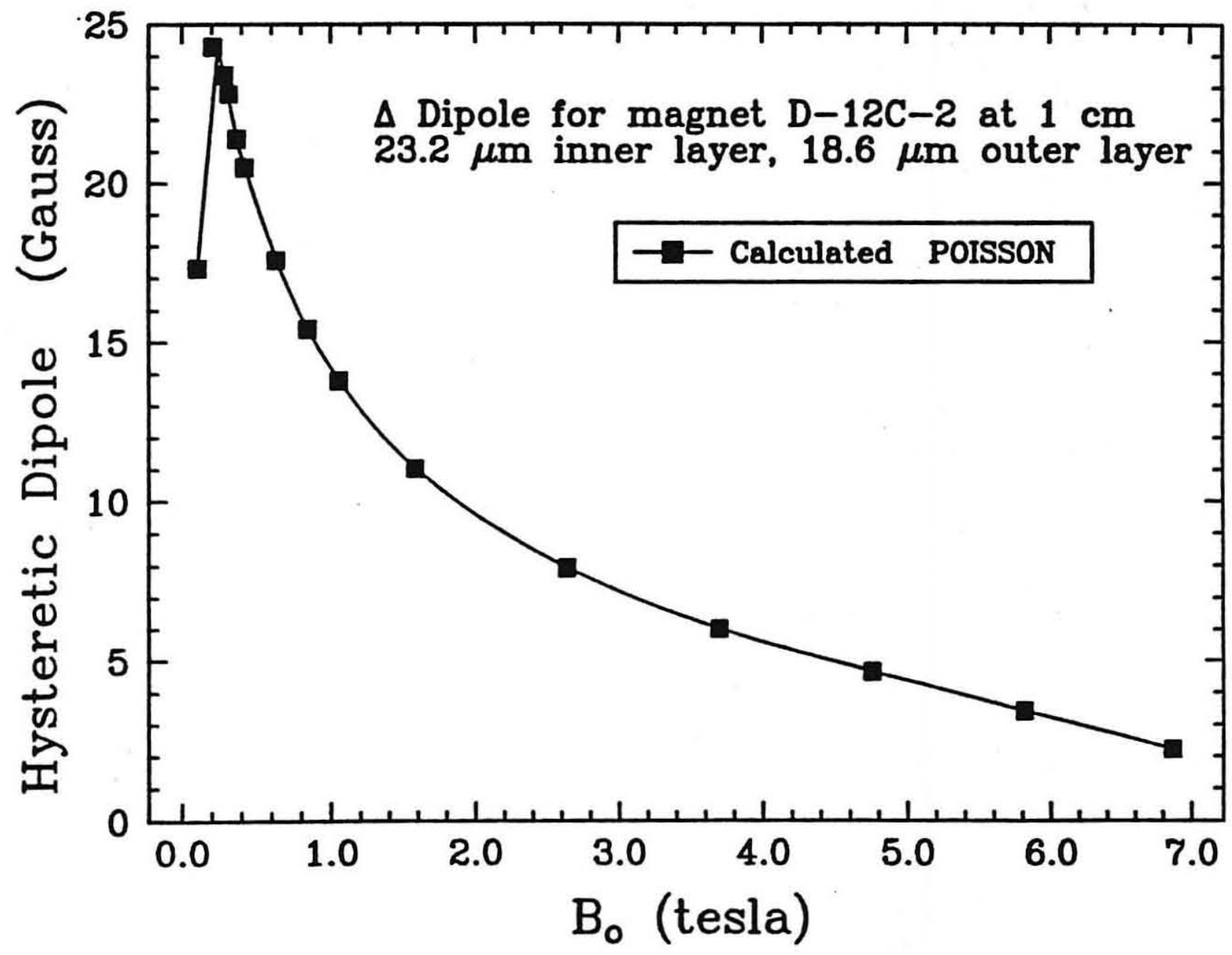

XBL 858-3192

Fig. 2. Differential dipole component (up minus down) due to magnetization. 
$\Delta$ Sextupole for design $\mathrm{D}(4 \mathrm{~cm})$

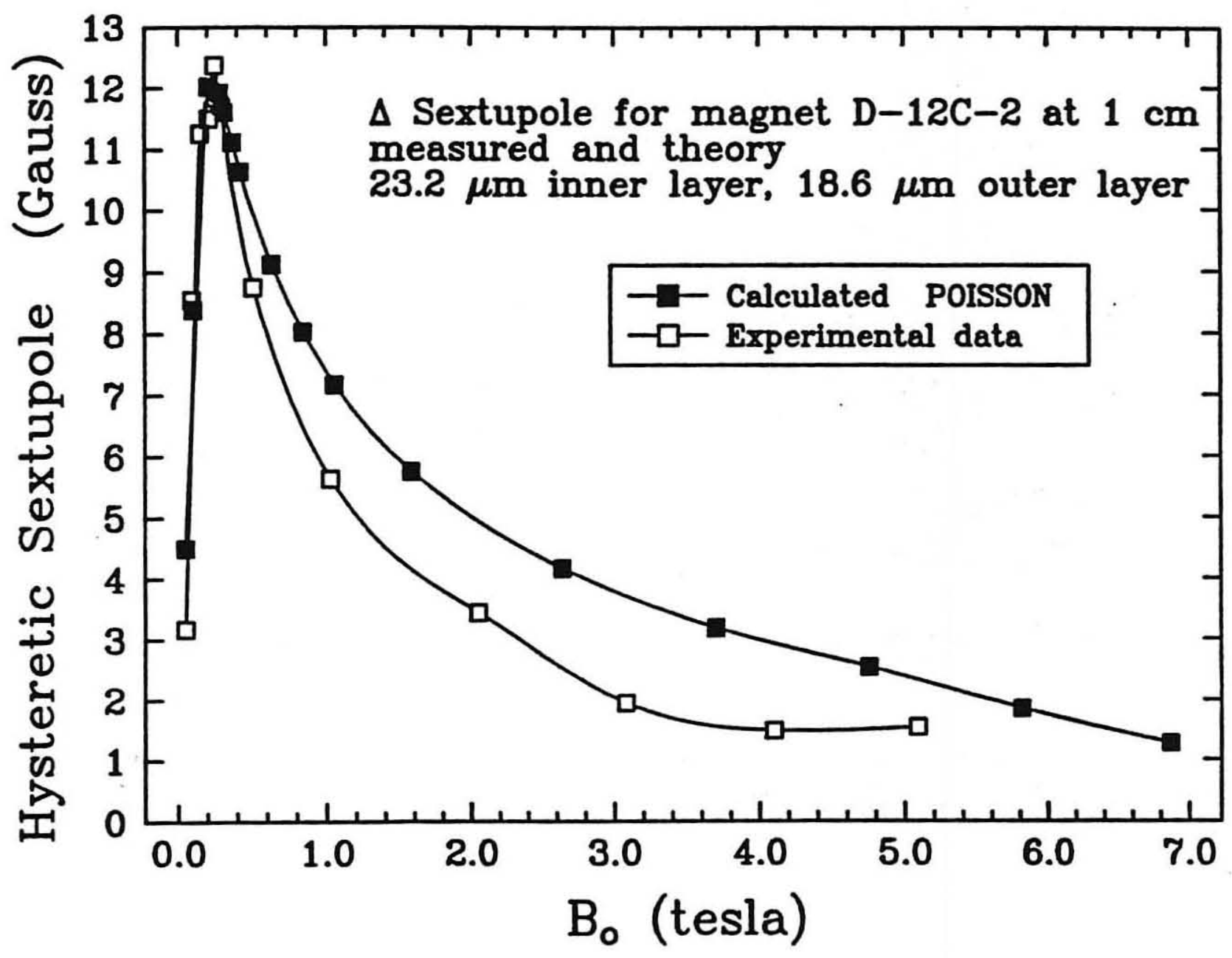

XBL $858-3189$

Fig. 3. Differential sextupole component due to magnetization. 
$\Delta$ Decapole for design $\mathrm{D}(4 \mathrm{~cm})$

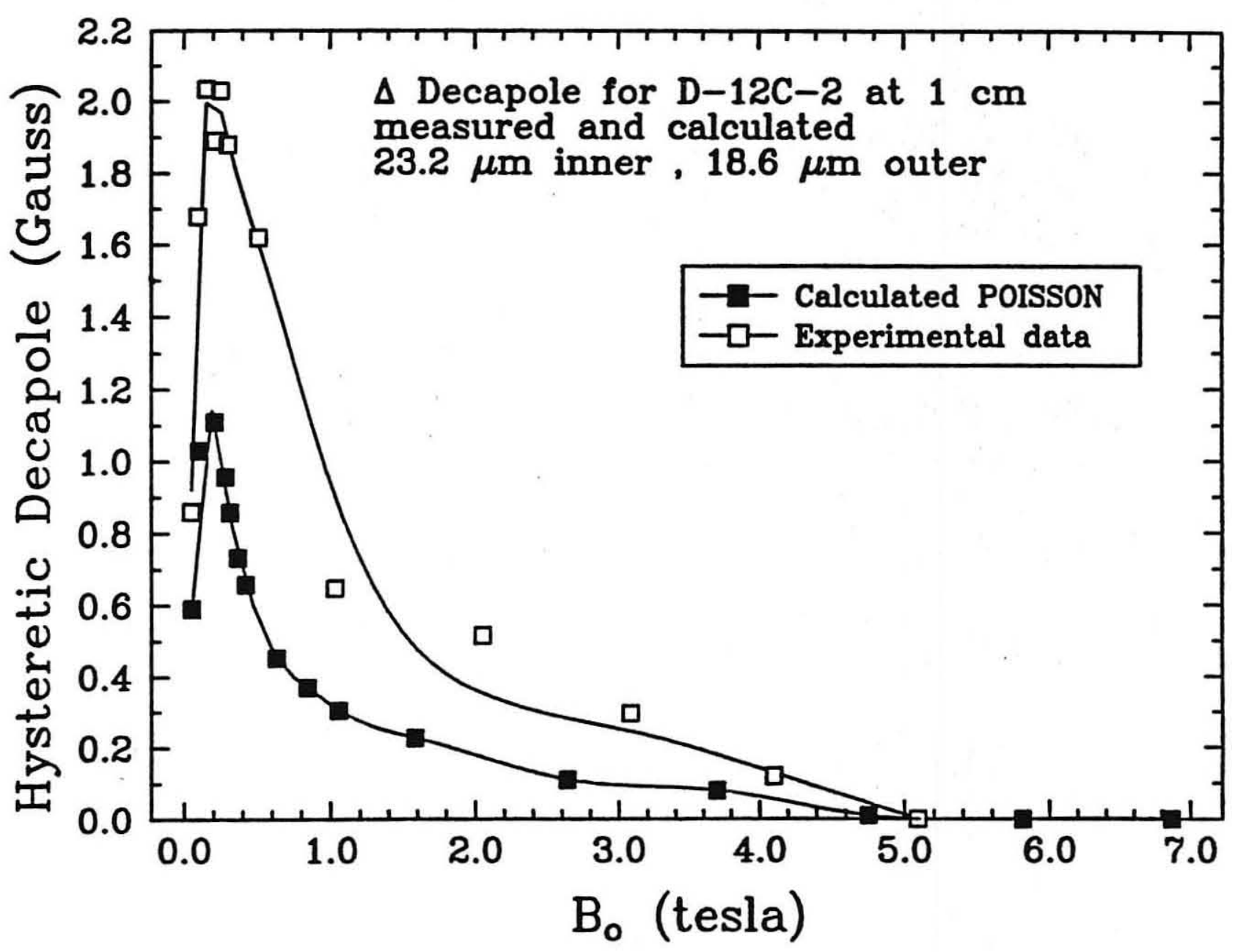

XBL $858-3193$

Fig. 4. Differential decapole component due to magnetization. 


\section{D-12C-2 Sextupole}

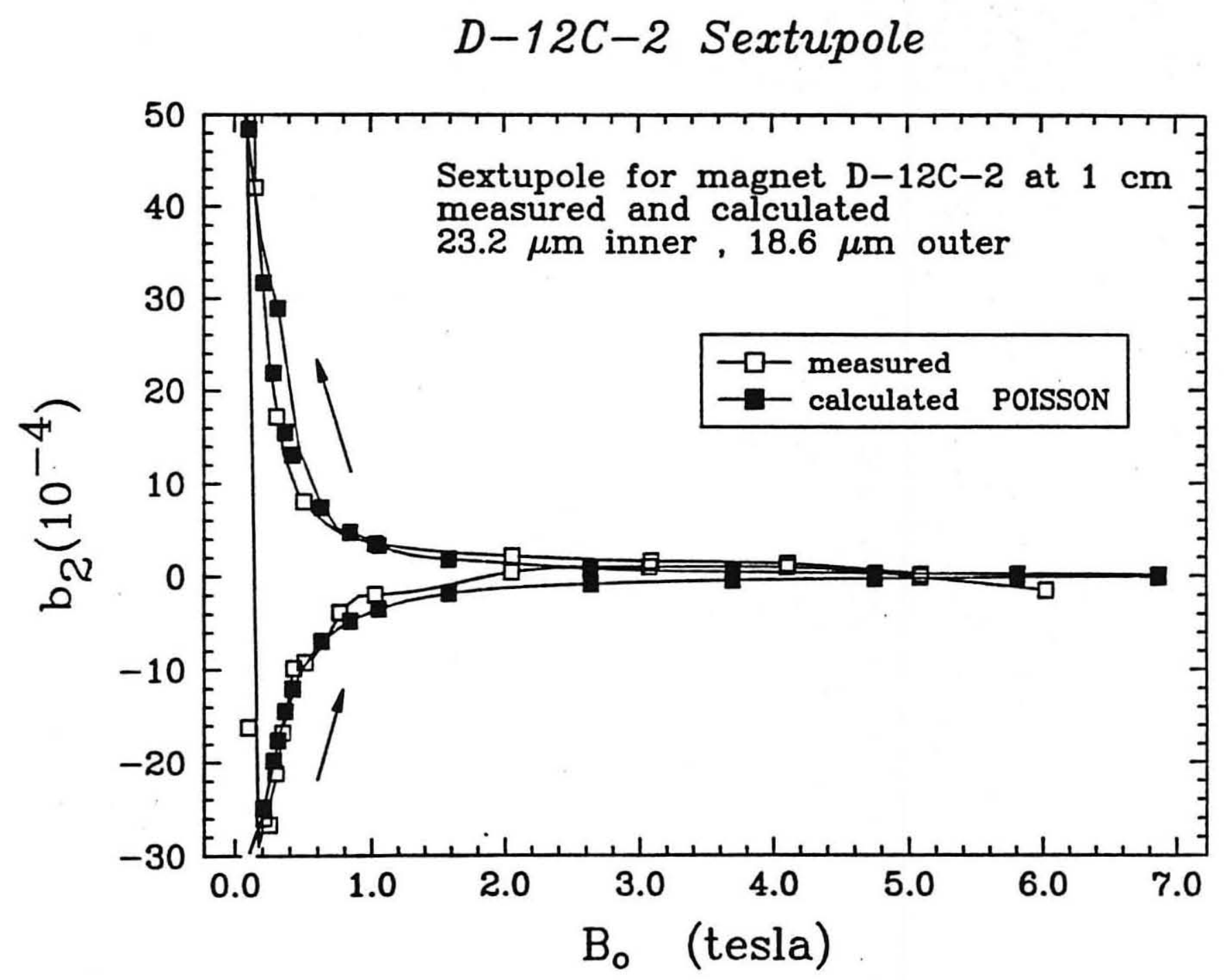

XBL 858-3191

Fig. 5. Sextupole coefficient due to magnetization during a full field cycle. 


\section{D-12C-2 Decapole}

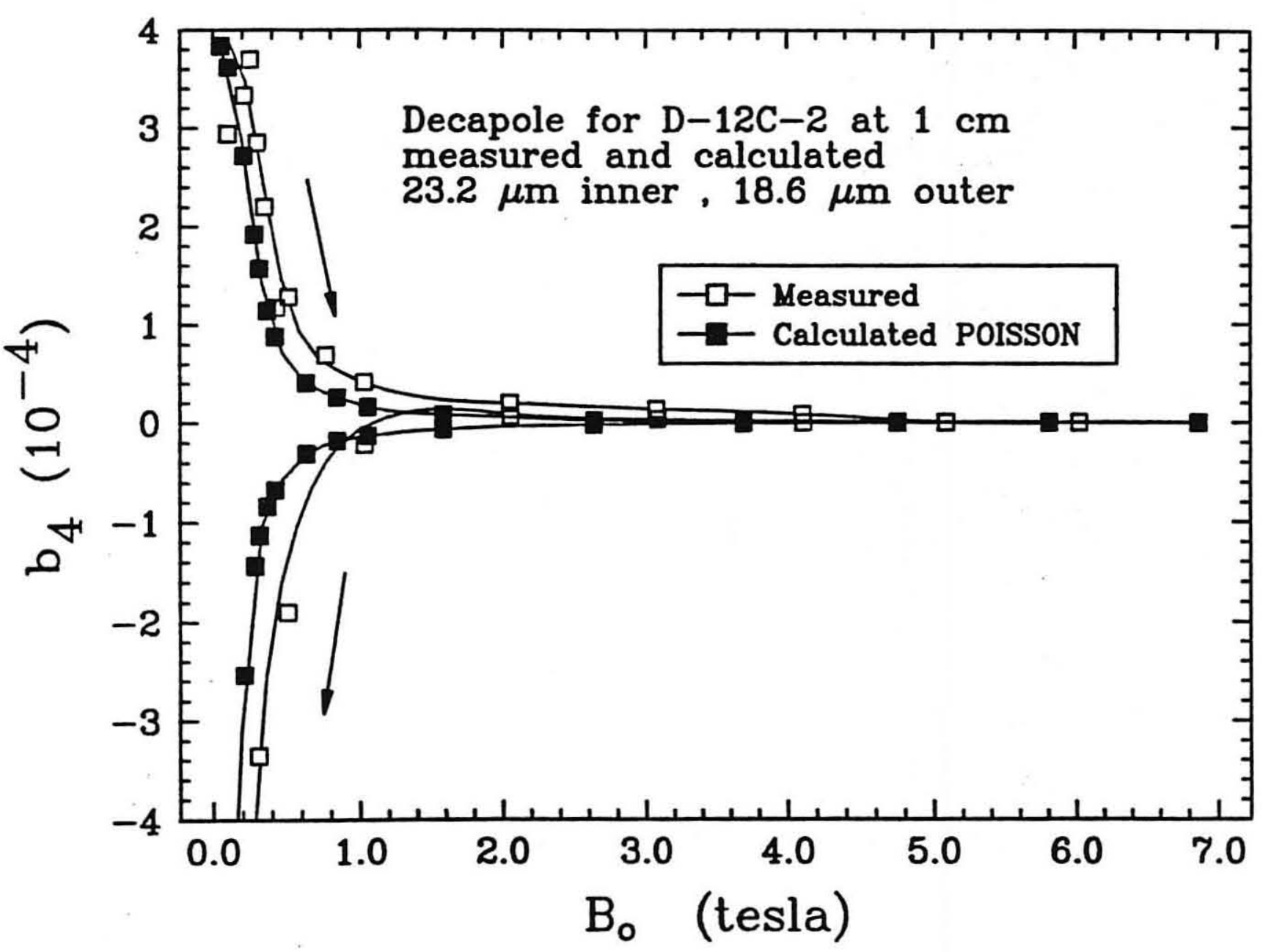

XBL $858-3190$

Fig. 6. Decapole coefficient due to magnetization during a full field cycle. 


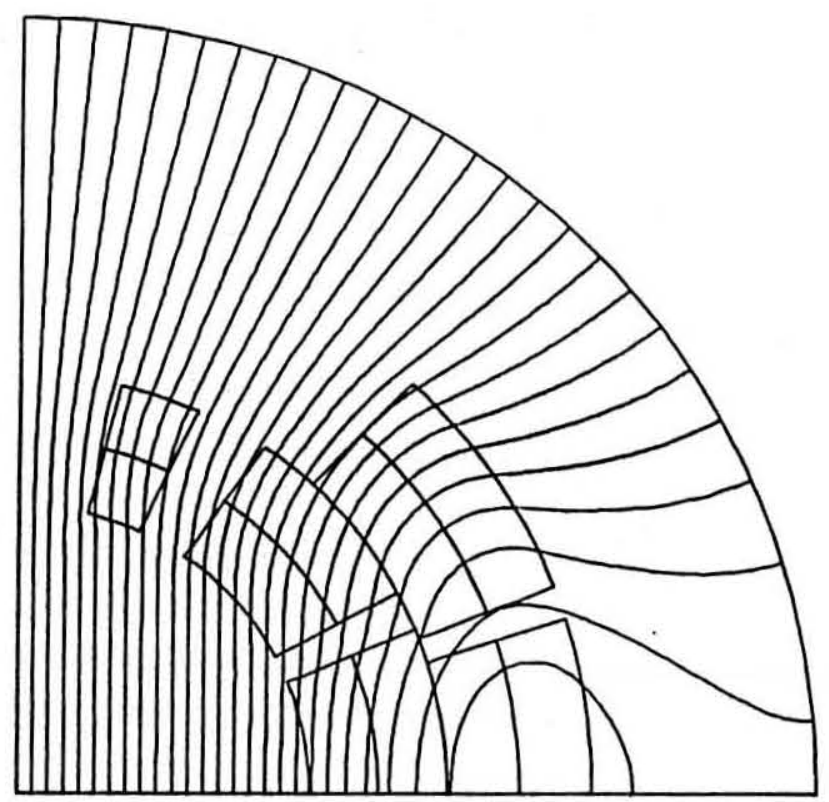

(a)

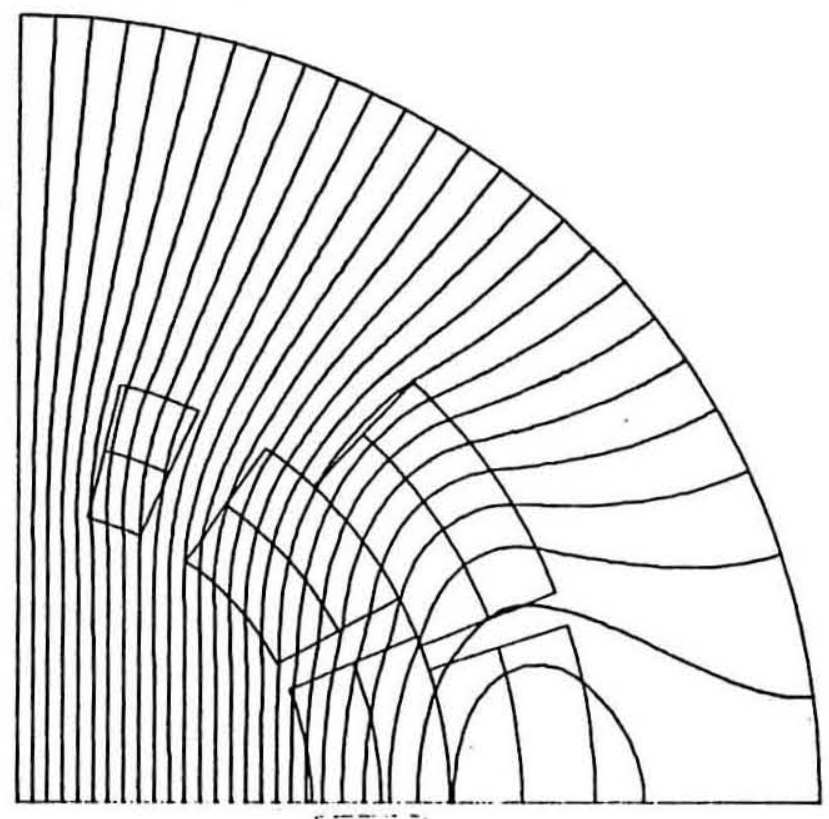

(b)

XBL $858-3195$ Fig. 7. Flux lines during a current increase (a) and a current decrease (b), at $0.28 \mathrm{~T}$.
The effect of magnetization can scarcely be distinguished. 

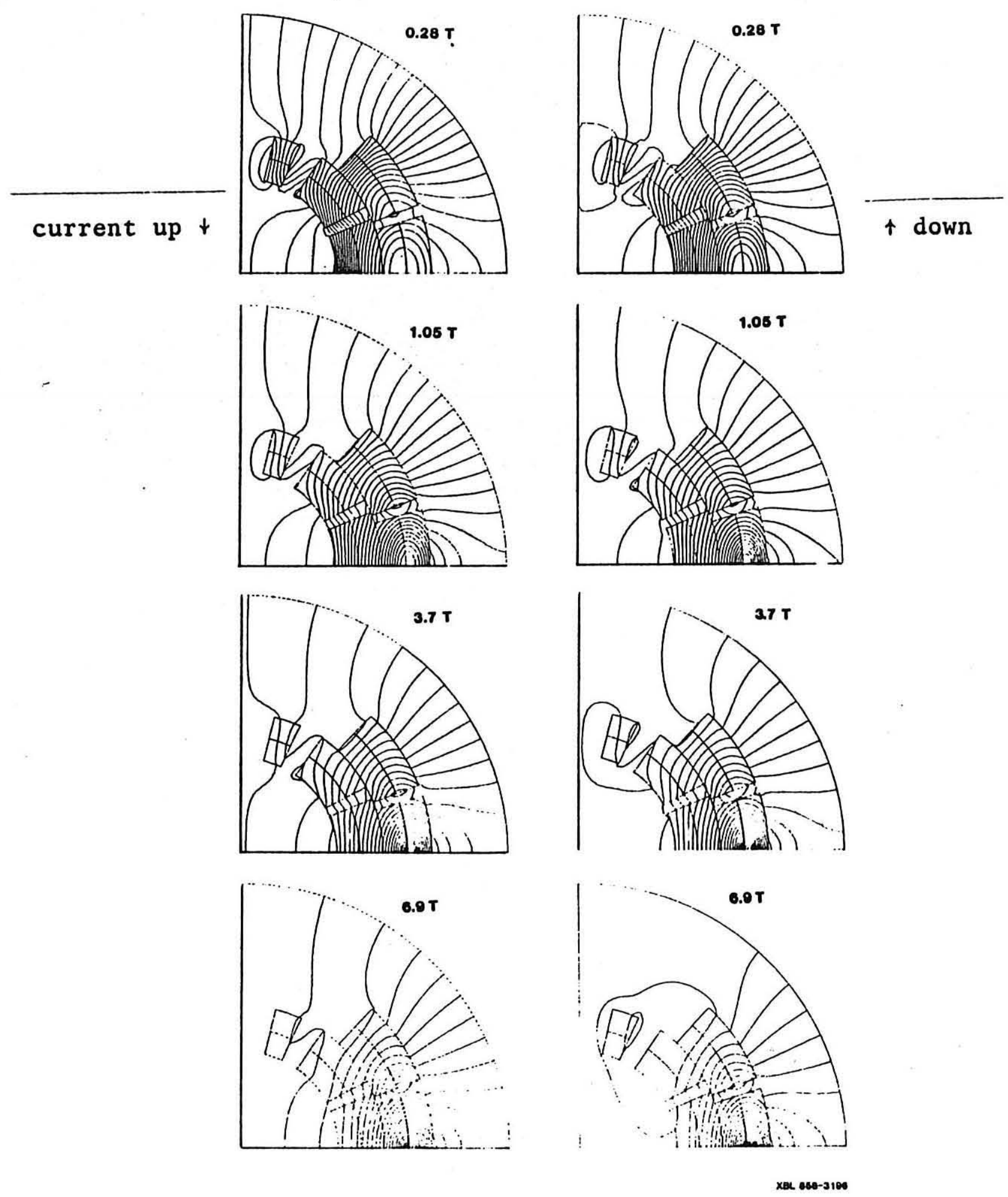

Fig. 8. Flux lines due to magnetization only, at various field levels, produced by subtracting the vector potential which includes magnetization from the one that has zero magnetization. 


\section{APPENDIX A}

\section{Scaling Procedure}

We shall be using the notation $F_{i j}$, where 1 corresponds to the type of scaling and $\mathbf{f}$ denotes the sublayer number.

1) Calculate $F_{1 j}$, the ratio between the true copper and superconductor cross section area to that of the winding block. The amount of copper Is as specifled by the Cu/sc ratio for each sublayer.

2) Generate the critical current curve as a function of fleld and superpose the load line corresponding to the field at the windings. From the ratio \& between transport current and critical current (at a given field) calculate $F_{2 j}=1-\delta$. It has been assumed that this curve is the same for all sublayers. Note that below 1.0 T, $F_{2 j}$ ls substantially constant ( $\propto 1.0)$ and is independent of field, therefore B or $H$ can be used as an independent variable; the distinction between $B$ and $H$ accordingly is not critical for describing the functional dependence of $F_{2 j}$.

3) Use $F_{3 j}$ to scale for varlations In fllament size. Thls is the ratio between fllament dlameters.

Once scaling factors for each of the sublayers have been generated they should be consolidated into a single factor according to:

$$
F_{j}=\prod_{j=1}^{3} F_{i j}
$$


The single original $M-H$ curve can now be scaled into four different curves, MF vs. $H_{\text {, }}$ each corresponding to an individual sublayer.

Inner-Layer - SC \#280

Filament diameter:

$$
d_{f}=23.2(\mu m) \quad(S C \text { only) }
$$

Number of fllaments:

$$
N_{f}=527
$$

Number of strands:

$$
N_{S}=23
$$

$$
R=1.3: 1 \mathrm{Cu} / \mathrm{sc}
$$

Strand current density at $5 \mathrm{~T}$ is:

$$
J_{C} \simeq 2200 \mathrm{~A} / \mathrm{mm}^{2}
$$

The strand diameter is:

$$
\begin{gathered}
D_{S}=d_{f} \sqrt{N_{f}(1+R)} \\
D_{S}=8.077 \times 10^{-4}(\mathrm{~m})=31.8(\mathrm{mi1})
\end{gathered}
$$

Strand cross section area:

$$
A_{S}=\frac{\pi D_{S}^{2}}{4}=5.124 \times 10^{-3}\left(\mathrm{~cm}^{2}\right)
$$

Cable cross section area (including $\mathrm{Cu}$ ):

$$
A_{c}=N_{s} \cdot A_{s}=0.117847\left(\mathrm{~cm}^{2}\right)
$$

\section{Quter-Layer - SC \#293}

Filament diameter:

$$
d_{f}=18.6(\mu \mathrm{m})
$$

Number of filaments:

$$
N_{f}=434
$$


Number of strands:

$$
\begin{gathered}
N_{S}=30 \\
R=1.8: 1 \mathrm{Cu} / \mathrm{sc}
\end{gathered}
$$

Strand current density at $5 \mathrm{~T}$ is:

$$
J_{c}=2400 \mathrm{~A} / \mathrm{mm}^{2}
$$

The strand diameter is:

$$
D_{S}=6.484 \times 10^{-4}(\mathrm{~m})=25.53(\mathrm{mil})
$$

and cross section area:

$$
A_{S}=3.302 \times 10^{-3}\left(\mathrm{~cm}^{2}\right)
$$

Cable cross section area (including Cu):

$$
A_{c}=0.09906\left(\mathrm{~cm}^{2}\right)
$$

$\underline{\underline{F 11}}$

Below is a table used to calculate the scale factor $F_{1 j}$ associated with the cross sectional area ratio between the copper + superconductor and the block conductor area (see Fig. I in the main text).

$\underline{\underline{F_{11}}}$

We have calculated the magnetization scale factor associated with transport current. We have scaled both layers based on the $J_{C}-B$ curve for the inner layer only. Plotted in Fig. 9 is the load line curve for magnet D-12C-2 and the cable critical current density curve (data for this curve were provided by short sample and magnetization measurements). 


\begin{tabular}{|c|c|c|c|c|c|c|}
\hline Sublayer & Location & $\begin{array}{l}\text { Block area } \\
A_{B}\left(\mathrm{~cm}^{2}\right)\end{array}$ & \# of ${ }_{\text {strands }}^{N_{s}^{\prime}}$ & $\begin{array}{c}\text { Area of Cu+sc }\left(\mathrm{cm}^{2}\right) \\
A_{S}^{\prime}=N_{S}^{\prime} A_{S}\end{array}$ & $A_{S}^{\prime} / A_{B}$ & $F_{1 j}$ \\
\hline 1 & 1 & 0.4313 & 11.5 & 0.3535 & 0.820 & 0.825 \\
\hline 1 & 2 & 0.5151 & 11.5 & 0.41246 & 0.8007 & 0.825 \\
\hline 1 & 3 & 0.2064 & 11.5 & 0.17677 & 0.85644 & 0.825 \\
\hline 2 & 1 & 0.5225 & 11.5 & 0.3535 & 0.6766 & 0.680 \\
\hline 2 & 2 & 0.6240 & 11.5 & 0.41246 & 0.6610 & 0.680 \\
\hline 2 & 3 & 0.2502 & 11.5 & 0.17677 & 0.7065 & 0.680 \\
\hline 3 & 1 & 0.5115 & 15 & 0.39624 & 0.7746 & 0.790 \\
\hline 3 & 2 & 0.7330 & 15 & 0.59436 & 0.811 & 0.790 \\
\hline 4 & 1 & 0.5913 & 15 & 0.39624 & 0.67011 & 0.680 \\
\hline 4 & 2 & 0.8471 & 15 & 0.59436 & 0.7016 & 0.680 \\
\hline
\end{tabular}


Magnet $D-12 C-2$

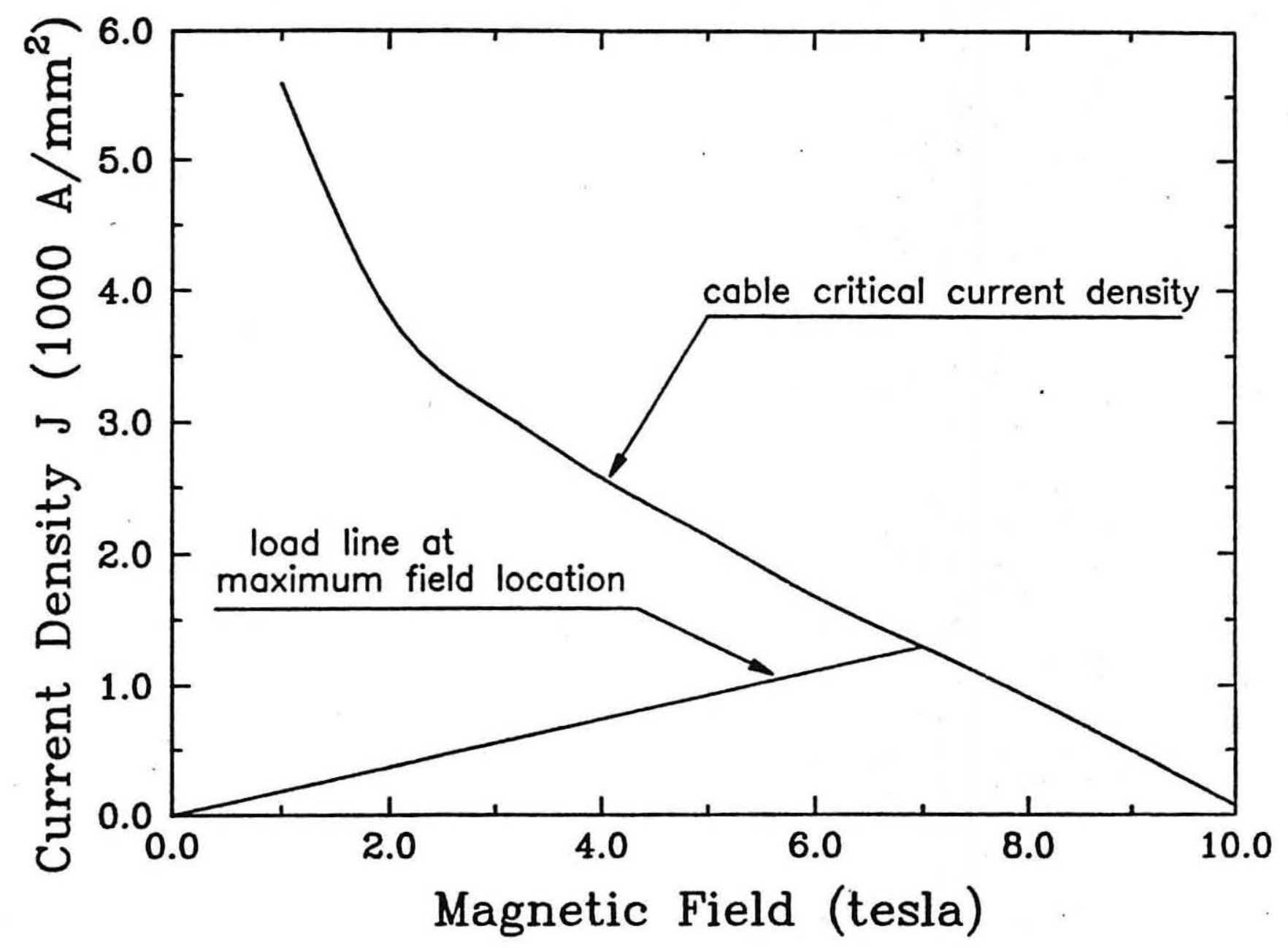

XBL 858-3194

Fig. 9. Critical current density curve for inner-layer cable. 


\begin{tabular}{l|l|l|l|l}
$B(T)$ & $I_{S S}^{(A)}$ & $I_{t}^{(A)}$ & $\delta=\frac{I_{t}}{I_{S S}}$ & $F_{2 J}=1-\delta$ \\
\hline 0.5 & 42000 & 473 & 0.0112 & 0.988 \\
1.0 & 32000 & 945 & 0.02937 & 0.9706 \\
2.0 & 21110 & 1891 & 0.08957 & 0.9104 \\
3.0 & 17216 & 2837 & 0.1648 & 0.835 \\
4.0 & 13988 & 3782 & 0.27 & 0.7296 \\
5.0 & 11273 & 4728 & 0.4194 & 0.5806 \\
6.0 & 8557 & 5673 & 0.6629 & 0.337 \\
7.0 & 6605 & 6605 & 1.0 & 0
\end{tabular}

We have plotted $F_{2 j}$ as a function of $B$ in Fig. 10 and used the following curve fit.

$$
\begin{aligned}
F_{2 j}= & 1-\delta=-5.806 \times 10^{-4} B^{4}+5.06 \times 10^{-3} B^{3}-2.564 \times 10^{-2} B^{2} \\
& -1.3295 \times 10^{-2} B+1.0003 .
\end{aligned}
$$

Errors in $F_{2 j}$ are of the order of $4 \%$.

We have scaled $J_{C}$ at low $B$ and $M$ at high $B$ using the relation:

$$
\begin{gathered}
2 \mu_{0} M=2 \mu_{0} \frac{2}{3 \pi} \lambda J_{c}(H) d \\
d \equiv \text { filament diameter } \\
\lambda \equiv \text { volume fraction of } S C ; \quad I /(1+R) \\
2 \mu_{0} M=M^{\text {up }}-M^{\text {down }}
\end{gathered}
$$




\section{Transport current correction}

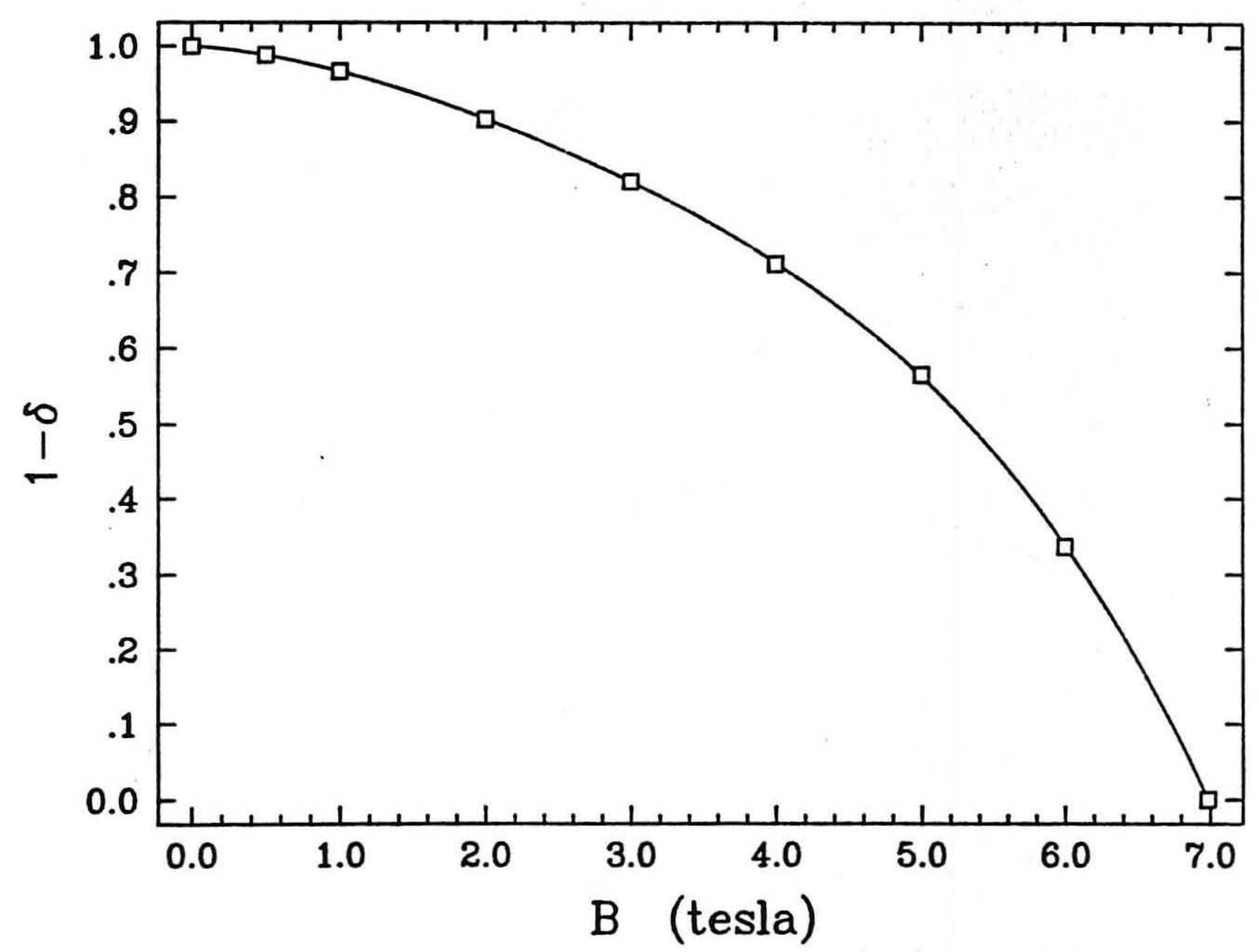

XBL $858-3188$

Fig. 10. Magnetization scaling factor due to transport current. 


\begin{tabular}{c|c|c|c|c|c}
$B(T)$ & $j_{C}\left(A / m^{2}\right)$ & $\begin{array}{l}\text { Calculated } \\
2 \mu_{0} M(\mathrm{mT})\end{array}$ & 2 measured $(\mathrm{mT})$ & $\frac{\text { measured }}{\text { calculated }}$ & Extrapolated (mT) \\
\hline 1.0 & $5590 \pm 12 \%$ & $30.85 \pm 3.8$ & 27.0 & 0.875 & 27.0 \\
2.0 & $3810 \pm 8 \%$ & $21.02 \pm 1.7$ & 18.8 & 0.894 & 18.8 \\
3.0 & $3095 \pm 8 \%$ & $17.08 \pm 1.5$ & 15.0 & 0.88 & 15.0 \\
4.0 & $2565 \pm 6 \%$ & $14.15 \pm 0.92$ & & & 12.45 \\
5.0 & $2125 \pm 3 \%$ & $11.72 \pm 0.41$ & & & 10.31 \\
6.0 & 1670 & 9.21 & & & \\
7.0 & 1290 & 7.12 & & & 6.10
\end{tabular}

$\underline{\underline{31}}$

This scaling factor reflects variations in fllament diameter. Since the measured magnetization curve of the inner layer was used, we have $F_{3_{\text {inner }}}=1.0$. In scaling the outer layer (18.6 $\mu \mathrm{m}$ filament diameter) from a $23.2 \mu \mathrm{m}$ fllament diameter of the inner layer we have $F_{3}$ outer $=18.6 / 23.6=0.802$.

Input to POISSON

A suitable input to POISSON requires a $B-H$ or $B-\gamma$ table $(\gamma=1 / \mu)$. We have decided on a $\mathrm{B}-\boldsymbol{\gamma}$ table. For the original $\mathrm{M}-\mathrm{H}$ curve we can write:

$$
B=H+M
$$

or

$$
B=\left(1+\frac{M}{H}\right) \quad H \text {. }
$$

With

$$
\mu_{o r}=1+\frac{M}{H} ; \quad \text { (or }=\text { original) }
$$


ORIGINAL MAGNETIZATION TABLE FROM GHOSH $23.2 \mu \mathrm{m} 1.3: 1$ SC

$\underline{\mathrm{UP}}$

$H$ (Gauss) $\quad \gamma=\frac{1}{\mu}$

\begin{tabular}{ll}
\hline 21.8 & .047 \\
43.6 & 0.104 \\
148.0 & 0.3229 \\
292.0 & 0.5835 \\
476.0 & 0.823 \\
580.0 & 0.9868 \\
702.7 & 1.1218 \\
890.0 & 1.1793 \\
1139.0 & 1.2050 \\
1414.0 & 1.2048 \\
1580.0 & 1.2018 \\
1846.0 & 1.17519 \\
2029.0 & 1.1621 \\
2243.0 & 1.1421 \\
2544.7 & 1.1183 \\
2815.0 & 1.10096 \\
3199.0 & 1.084 \\
3766.9 & 1.064 \\
4395.4 & 1.0495 \\
5220.0 & 1.03829 \\
6010.0 & 1.03088 \\
7010.0 & 1.02488 \\
8262.7 & 1.0197 \\
9166.0 & 1.016766 \\
9969.4 & 1.0146 \\
12219.7 & 1.0105 \\
14681.0 & 1.00796 \\
177.75 .8 & 1.005766 \\
21485.7 & 1.00446 \\
25846.0 & 1.003159 \\
30153.8 & 1.00249 \\
35358.2 & 1.00205 \\
40000.0 & 1.0015 \\
50000.0 & 1.0010 \\
60000.0 & 1.00067 \\
70000.0 & 1.00045 \\
&
\end{tabular}

DOWN

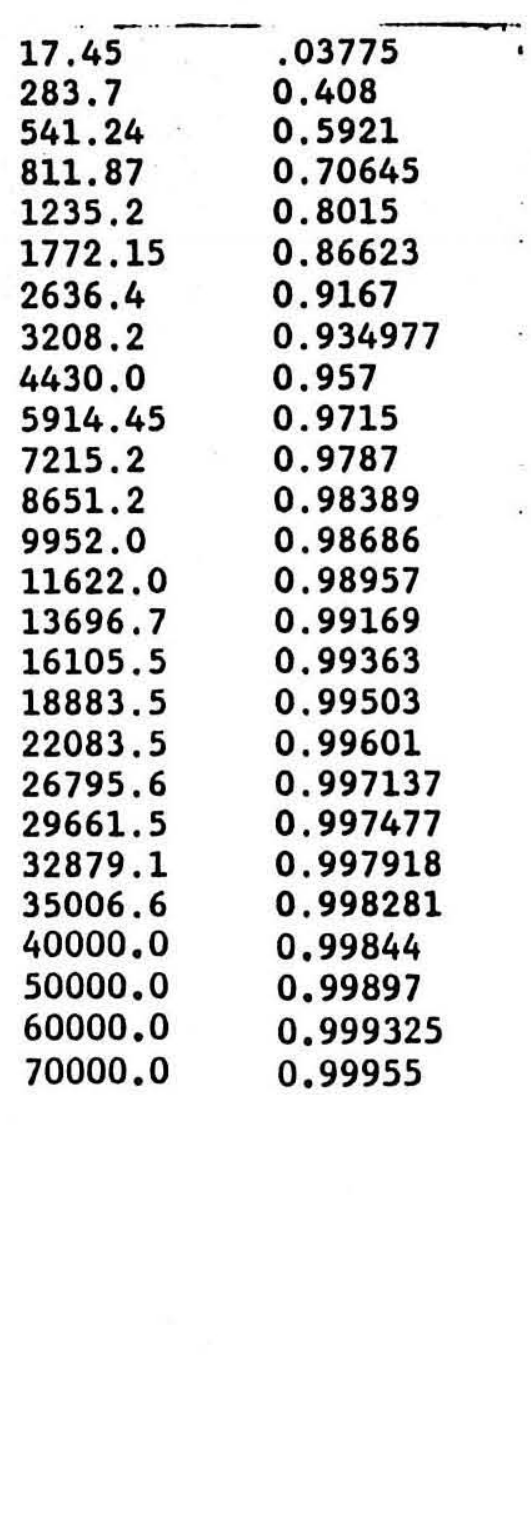




$$
B=\mu_{o r} H \text {. }
$$

The permeability of the scaled magnetization is written as:

$$
\mu_{s j}=1+\frac{M F_{f}}{H} ;
$$

or

$$
\mu_{s j}=1+\left(\mu_{o r}-1\right) F_{j} .
$$

In terms of $\gamma$ we have:

$$
r_{s j}=\frac{1}{1+\left(\frac{1}{r_{o r}}-1\right) F_{j}}
$$

and

$$
B=\left(\frac{1}{r_{s j}}\right) H
$$

Note that the parameters $\gamma_{S j}$ are $H$ dependent.

The $F_{j}$ calculated for the four sublayers are:

$$
\begin{array}{ll}
F_{1}=0.825 & F_{3}=0.633 \\
F_{2}=0.680 & F_{4}=0.545
\end{array}
$$

at low field strengths, but at higher flelds the factors $F_{2 j}$ that contribute to these quantities will introduce some field dependence. The original and scaled magnetization curves are included here both in tabular form, and as plotted curves, Fig. 11.

Several restrictions in input entries to POISSON are noted. 
Magnetization Curves $D-12 C-2$

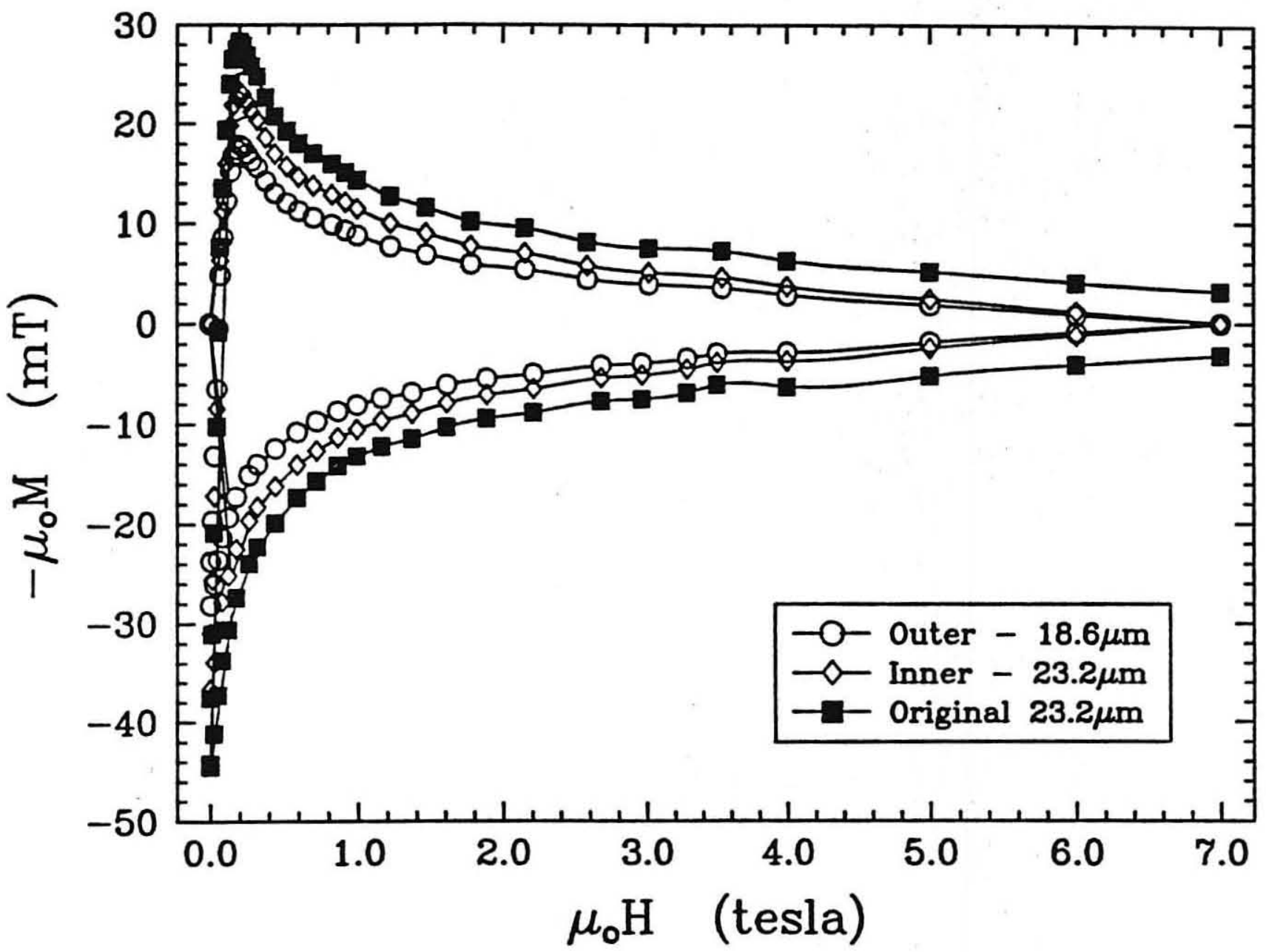

XBL 858-3187

Fig. 11. Some of the magnetization curves used in the present calculations. 
POISSON is restricted in the number of permeability tables it can accept. Excluding the built-in table, up to three additional tables can be entered. This condition can be relaxed through the use of the POISSON stacking factor. We decided, however, to limit magnetization entries in the present work to three tables and therefore substituted $F_{2}$ for $F_{3}$.

The second and the most troublesome entry to POISSON arises from following this technique at low flelds ( $H<300$ Gauss), where double values of $\gamma$ vs. B can then occur. Since POISSON does not support B entries in a permeability table which are - less than zero we were forced to approximate the table entries at low fields. An adjustment to the $\mathrm{M}-\mathrm{H}$ curve at low field (Fig. 12) was made so that at $\mathrm{H}=0, \mathrm{M}=0$ (Fig. 13). This was done by drawing a linear asymptote from the origin to the upper curve and adjusting the lower curve analogously by drawing a linear curve from the origin over the same field interval. 
Magnetization Curves D-12C-2

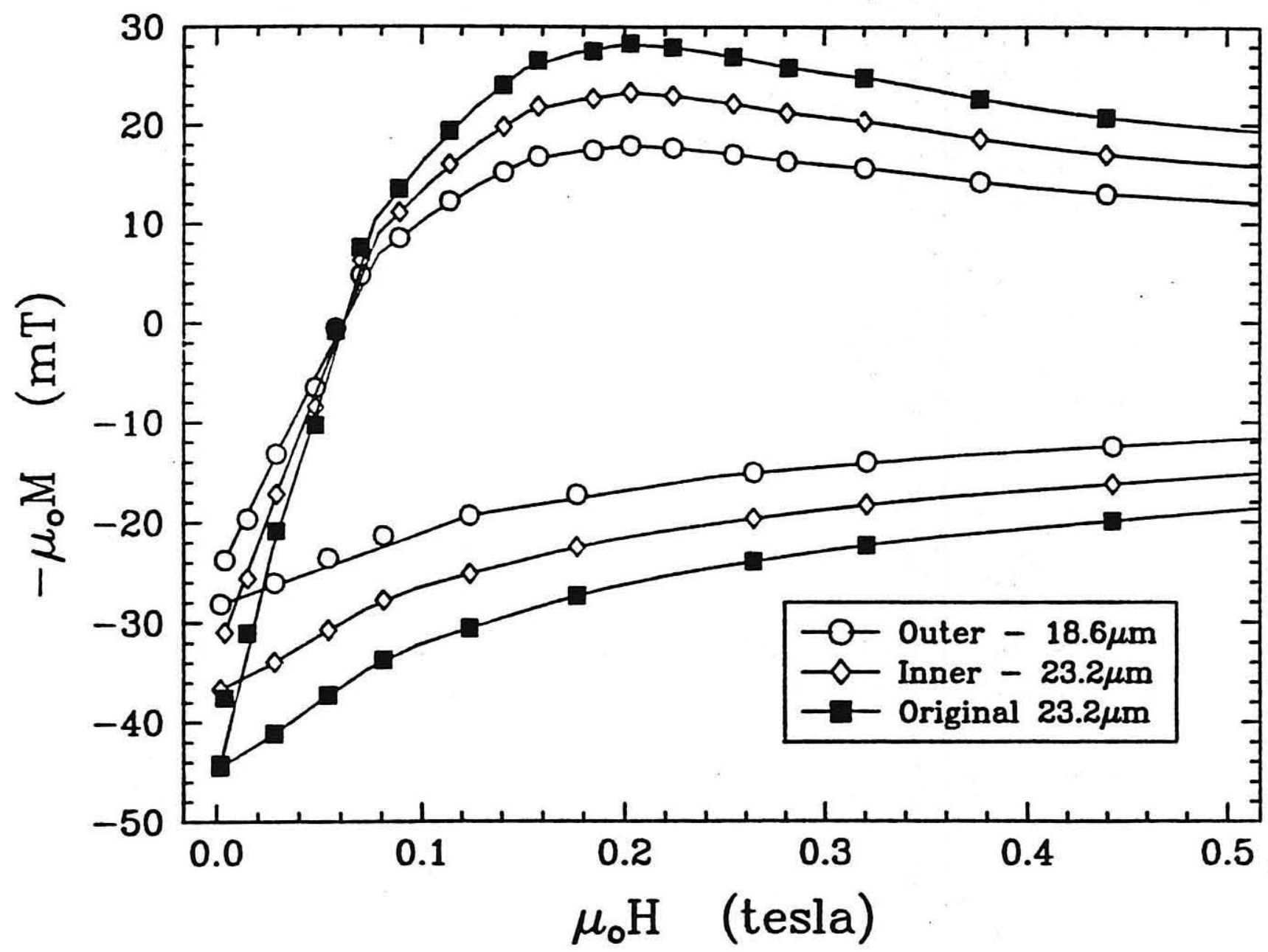

XBL $858-3186$

Fig. 12. The low field magnetization values as derived from scaling. 
Magnetization Curves $D-12 C-2$

As used in POISSON

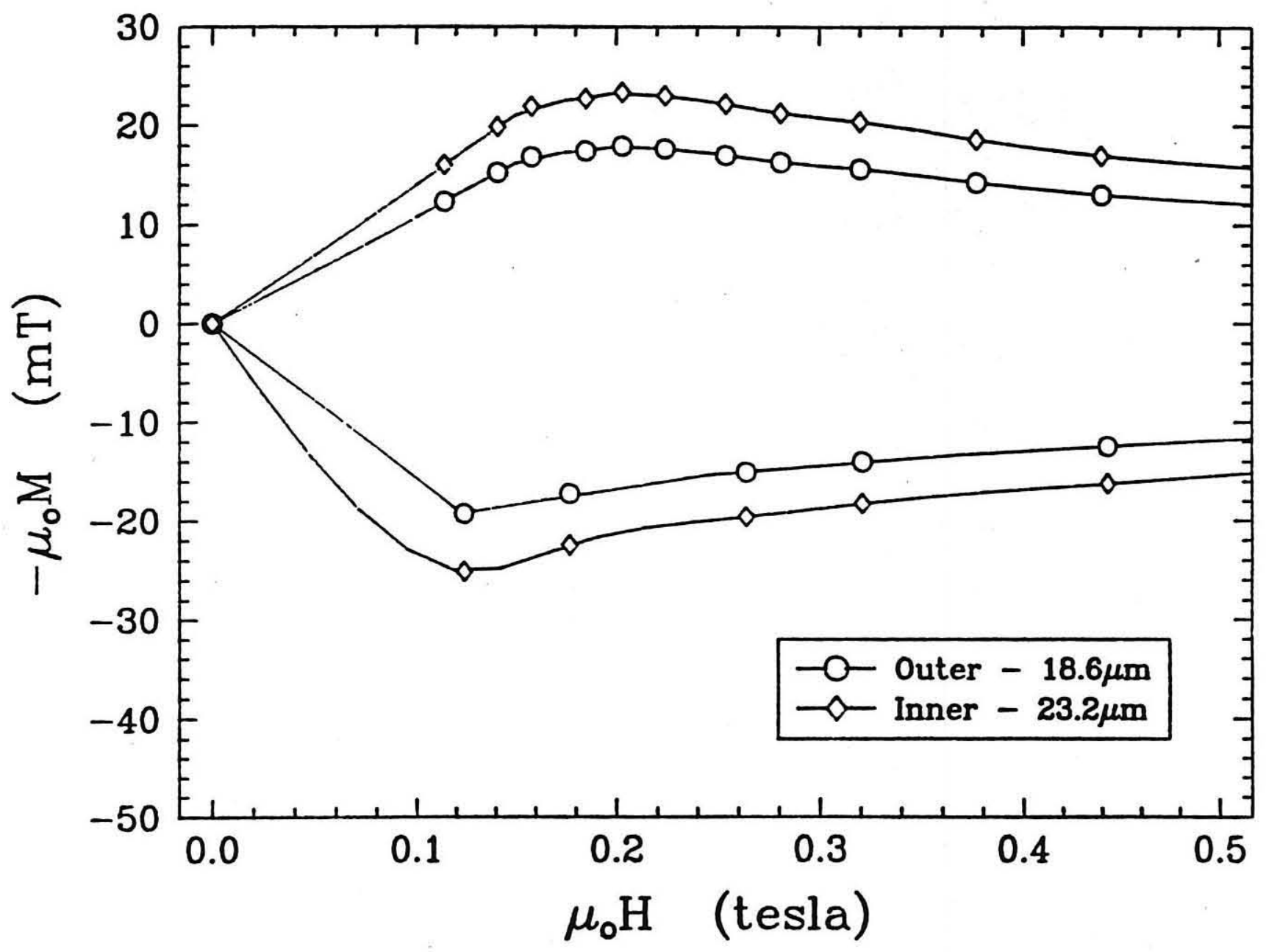

XBL 858-3185

Fig. 13. Low field magnetization adjustment as introduced to POISSON. 